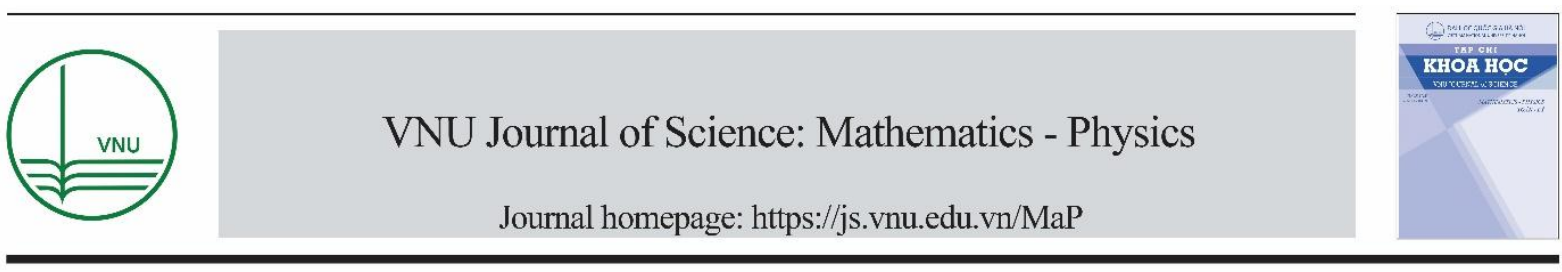

Original Article

\title{
Titanate Tubular Loaded Diatom Fabricated by a Facial Hydrothermal Method for Photocatalytic Enhancement under Visible Light Irradiation
}

\author{
Nguyen Xuan Sang ${ }^{1, *}$, Vo Cao Minh ${ }^{2}$, Le Hong Phuc ${ }^{3}$, \\ Nguyen Thi Quynh Trang ${ }^{2}$, Vo Quang $\mathrm{Mai}^{4}$ \\ ${ }^{1}$ Department of Electronics and Telecommunication, Saigon University, \\ 273 An Duong Vuong, Ward 3, District 5, Ho Chi Minh City, Vietnam \\ ${ }^{2}$ Department Environmental Science, Saigon University, 273 An Duong Vuong, \\ Ward 3, District 5, Ho Chi Minh City, Vietnam \\ ${ }^{3}$ Department of Novel and Nanostructure of Materials, Ho Chi Minh City Institute of Physics, Vietnam \\ Academy of Science and Technology, Ho Chi Minh City, Vietnam \\ ${ }^{4}$ Department Natural Sciences Education, Saigon University, 273 An Duong Vuong, \\ Ward 3, District 5, Ho Chi Minh City, Vietnam \\ Received 19 March 2020 \\ Revised 20 April 2020; Accepted 15 June 2020
}

\begin{abstract}
In this study, homogeneous titanate tubular (TNT) and diatom/TNT composites (D/TNT) were prepared by a facile hydrothermal method. The crystalline structure and morphology of the synthesised samples were studied by X-ray diffractometry (XRD), Raman spectroscopy, Transmission Electron Microscopy (TEM). Furthermore, the photocatalytic activity for degradation of methylene blue (MB) was investigated under sunlight irradiation. The results showed that the MB degradation by D/TNT was higher than bare TNT. Optical properties of the synthesized materials with enhanced photocatalytic activity could be explained through both UVvis diffuse absorption and photoluminescence emission measurement. These results indicated that there was bandgap narrowing and longer carrier lifetime in the composite sample.

Keywords: TNT, diatom, hydrothermal method, photocatalytic activity.
\end{abstract}

\footnotetext{
${ }^{*}$ Corresponding author.

Email address: sangnguyen@sgu.edu.vn
}

https//doi.org/ 10.25073/2588-1124/vnumap.4493 


\section{Introduction}

At present, industries thrive to keep up with human needs, textile and dyeing industry is not out of the trend. Organic ingredients in textile wastewater are very difficult to biodegrade and toxic to the environment [1]. Consequently, scientists around the world are studying to find ways to handle organic components in wastewater [2]. $\mathrm{TiO}_{2}$ nanomaterials with tubular structure (TNT) have shown superiority in the treatment of textile wastewater by photocatalytic ability [3]. However, TNT has a large bandgap which does not consume visible light effectively for the photocatalytic application. Many studies have been carried out to reduce the bandgap and, thus enhance photocatalytic ability [45]. Diatoms, i.e. phytoplankton, are popular in many parts of the world such as the sea, fresh water [6]. Diatom has a natural porous structure, which can spread light by diffraction processes and can absorb light within a broad range. With these things, diatoms are very useful in photocatalytic activity by compositing with other photocatalysts [1, 6-7]. In this study, TNT was in-situ hydrothermally grown with diatom.

Herein the synthesized samples were characterized by transmission electron microscopy (TEM), energy dispersive X-ray spectroscopy (EDX), X-ray diffractometry (XRD), Raman spectroscopy, UVvis diffuse absorption spectra and photoluminescence emission spectra (PL). Photocatalytic evaluation was examined by the degradation of methylene blue solution under direct sunlight exposure.

\section{Experimental}

\subsection{Chemicals}

The chemicals have been purchased and used without further purification: diatom (SigmaAldrich), $\mathrm{TiO}_{2}$ powder (Merck), sodium hydroxide $\left(\mathrm{NaOH}\right.$, Merck), acetone $\left(\mathrm{C}_{3} \mathrm{H}_{6} \mathrm{O}\right)$, ethanol $\left(\mathrm{C}_{2} \mathrm{H}_{6} \mathrm{O}\right)$, hydrochloric acid ( $\mathrm{HCl}$, China, 37\%), methylene blue $\left(\mathrm{C}_{16} \mathrm{H}_{18} \mathrm{CINS}_{{ }_{3}} \mathrm{H}_{2} \mathrm{O}\right.$, JHD Fine Chemicals, China, 99\%).

\subsection{Hydrothermal synthesis of TNT and D/TNT composite}

TNT material was synthesized by hydrothermal method in a $150 \mathrm{ml} 58 \mathrm{eflon}$-sealed stainless steel autoclave for $24 \mathrm{~h}$ at $135^{\circ} \mathrm{C}$ as described previously [5,8]. Typically, firstly, $34 \mathrm{~g} \mathrm{NaOH}$ was dissolved in $78 \mathrm{ml}$ DI water by magnetic stirring for $15 \mathrm{~min}$. Then, $0.84 \mathrm{~g} \mathrm{TiO}_{2}$ powder was added into the solution and was stirred for $15 \mathrm{~min}$. After that, the resulting mixture was poured into the autoclave and started the hydrothermal process by the oven. After the hydrothermal process had finished, the autoclave was cooled to room temperature. Next, $\mathrm{HCl}$ acid solution was added to the autoclave for 30 $\mathrm{min}$, then washed several times with DI water until a $\mathrm{pH}$ of 7 was reached. Then, it was in the oven for $6 \mathrm{~h}$ at $100^{\circ} \mathrm{C}$ until the completely dried powder was obtained. For the fabrication of diatom and TNT composite, the amount of diatom of $20 \mathrm{wt} . \%$ was added to the solution with magnetic stirring continuously in $10 \mathrm{~min}$ before pouring into the autoclave for the hydrothermal synthesis described above.

\section{Results and discussion}

\subsection{Transmission electron microscopy (TEM)}

In fig. 1, the TEM micrographs show morphological properties of TNT and D/TNT. The TNTs were appreared with homogenous diameter of about $\sim 8$ nanometers and several hundred nanometers in 
length as shown in Fig. 1a. In the composite sample, diatom and TNT were both appeared and TNT formed on diatom with the same diameter and length as pure TNT as shown in Fig. 1b. Based on the above result, both TNT and diatom appeared in the D/TNT composite, which showed the D/TNT composite with two regions including silica and titania [9]. In the treatment process of pollutants, silica region could adsorb the pollutants which were transported to the titania region by surface diffusion.
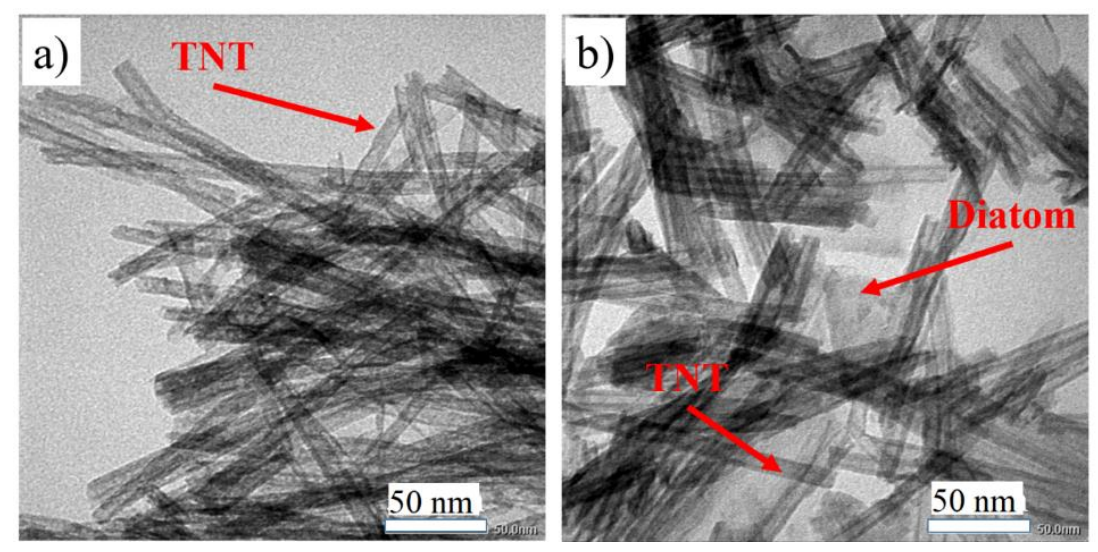

Figure 1. The TEM images of TNT and D/TNT composite

\section{2. $X$-ray diffraction patterns $(X R D)$}

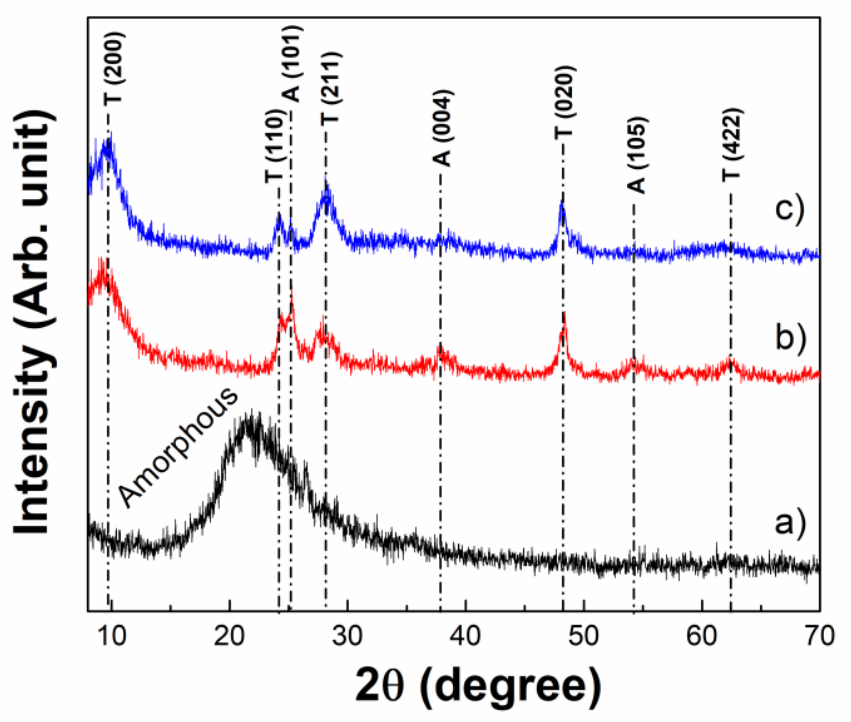

Figure 2. XRD patterns of diatom (a), TNT (b) and D-TNT composite (c).

The Fig. 2 shows the XRD patterns of diatom, TNT and D/TNT composite. The peaks at $9.88^{\circ}$, $24.17^{\circ}, 28.06^{\circ}, 48.14^{\circ}$ and $62.38^{\circ}$ for the TNT corresponding to the (200), (110), (211), (020) and 
(422) planes, respectively, were attributed to monoclinic structure of $\mathrm{H}_{3} \mathrm{Ti}_{2} \mathrm{O}_{7}$ [10-13]. In addition, there appeared anatase $\mathrm{TiO}_{2}$ peaks at $25.23^{\circ}, 38.15^{\circ}$ and $54.48^{\circ}$ corresponding to the (101), (004) and (105) planes, respectively [5,14-15]. On the XRD pattern of D/TNT composite, these appear the characteristic peaks similar to TNT. However, the intensity of these anatase peaks at $38.15^{\circ}, 54.48^{\circ}$ and $62.38^{\circ}$ are decreased indicating effect of diatom in the formation of TNT structure. The broad peak at $22.15^{\circ}$ for diatom due to its amorphous structure $[1,16]$.

\subsection{Raman scattering specctra}

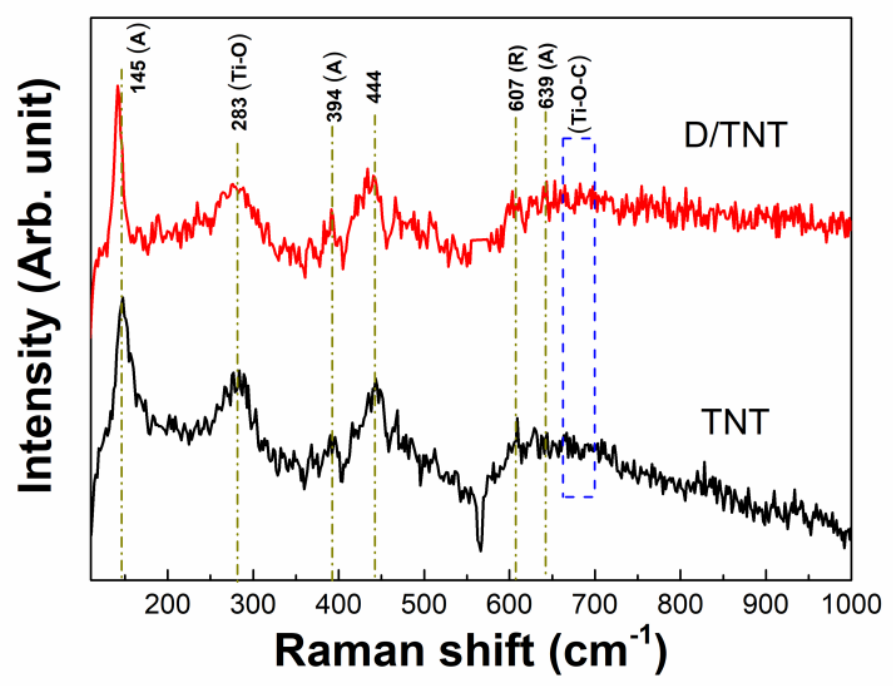

Figure 3. Raman spectra of TNT and D/TNT composite in the range of $110-1000 \mathrm{~cm}^{-1}$

Fig. 3 shows Raman spectra of TNT and D/TNT composite in the range of $110-1000 \mathrm{~cm}^{-1}$. For the TNT, it indicates that TNT has the characteristic peaks for $\mathrm{TiO}_{2}$ anatase phase at $145,394,639 \mathrm{~cm}^{-}$ ${ }^{1}$ and a characteristic peak of the rutile phase at $607 \mathrm{~cm}^{-1}[1,17-18]$. In addition, the peak at $283 \mathrm{~cm}^{-1}$ is assigned to Ti-O-Na bonds with layer structure [18-19]. Furthermore, Ti-O-C bonds are appeared in the range of $670-700 \mathrm{~cm}^{-1}$ [20]. Besides, the peak at $444 \mathrm{~cm}^{-1}$ locates at $\mathrm{E}_{\mathrm{g}}$ mode normally assigned to the characteristic peak of rutile phase [18]. However, in another study, the peak at $444 \mathrm{~cm}^{-1}$ is confirmed as the characteristic peak of the tubular titanate structure [13]. Raman spectrum of D/TNT composite indicates the vibration peaks similar to the vibration peaks of Raman spectrum of TNT. In general, based on the similarity of materials on XRD pattern and Raman spectra, the structure of TNT is not affected when TNT is composite with diatom.

\subsection{UV-vis absorption spectra}

Fig. 4a shows the UV-vis diffuse absorption spectra of TNT and D/TNT composite. There are several techniques to derive bandstructure of the semiconductor through this measurement [21-23]. One of the simple and useful methods is Tau's plot with Kubelka-Munk (KM) function. Figure 4b showed the bandgap of TNT and D/TNT by using the plot where the bandgap values of TNT and 
D/TNT are 3.55 and $3.41 \mathrm{eV}$, respectively. This is because when TNT is composited with diatom, the bandgap value of D/TNT composite is reduced. The reduction of the bandgap contributes to impoved the photocatalytic ability, because the excited electrons in composite materials transport from the valence band to the conduction band more easily with broader range [24].
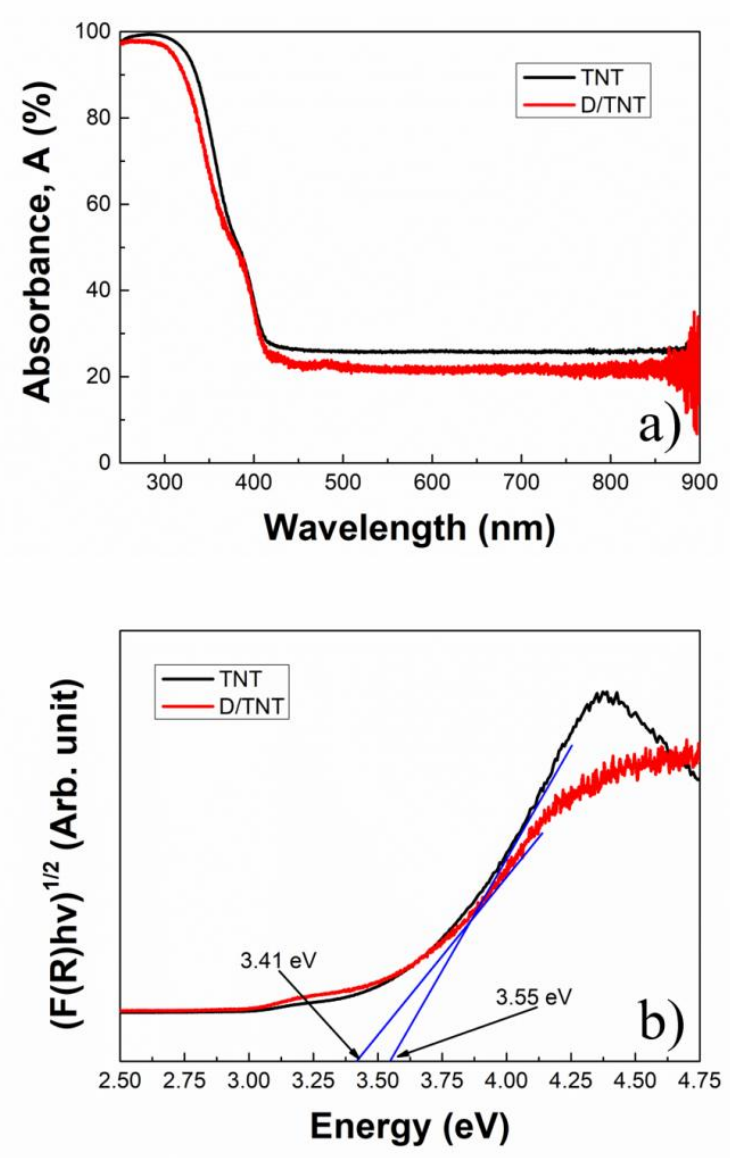

Figure 4. The UV-vis adsorption spectra of TNT and D/TNT samples (a), Bandgap values are determined by Tauc's plot (b)

\subsection{Photocatalytic activity investigation}

Fig. 5 shows sequence of physical absorption and photocatalytic ability of TNT and D/TNT with MB concentration of $25 \times 10^{-6} \mathrm{~mol} / \mathrm{l}$. TNT and D/TNT composite were surveyed simultaneously in the same conditions, the step of $\mathrm{Uv}-\mathrm{Vis}$ was $1 \mathrm{~nm}$ in the range of $450-800 \mathrm{~nm}$. The solution was extracted at 120 min to investigate the physical absorption ability of TNT and D/TNT composite. Then, from 120 to $300 \mathrm{~min}$, the solution was frequently extracted to investigate the photocatalytic ability of TNT and D/TNT composite. Photocatalytic process of TNT and D/TNT composite carried out under sunlight irradiation. 

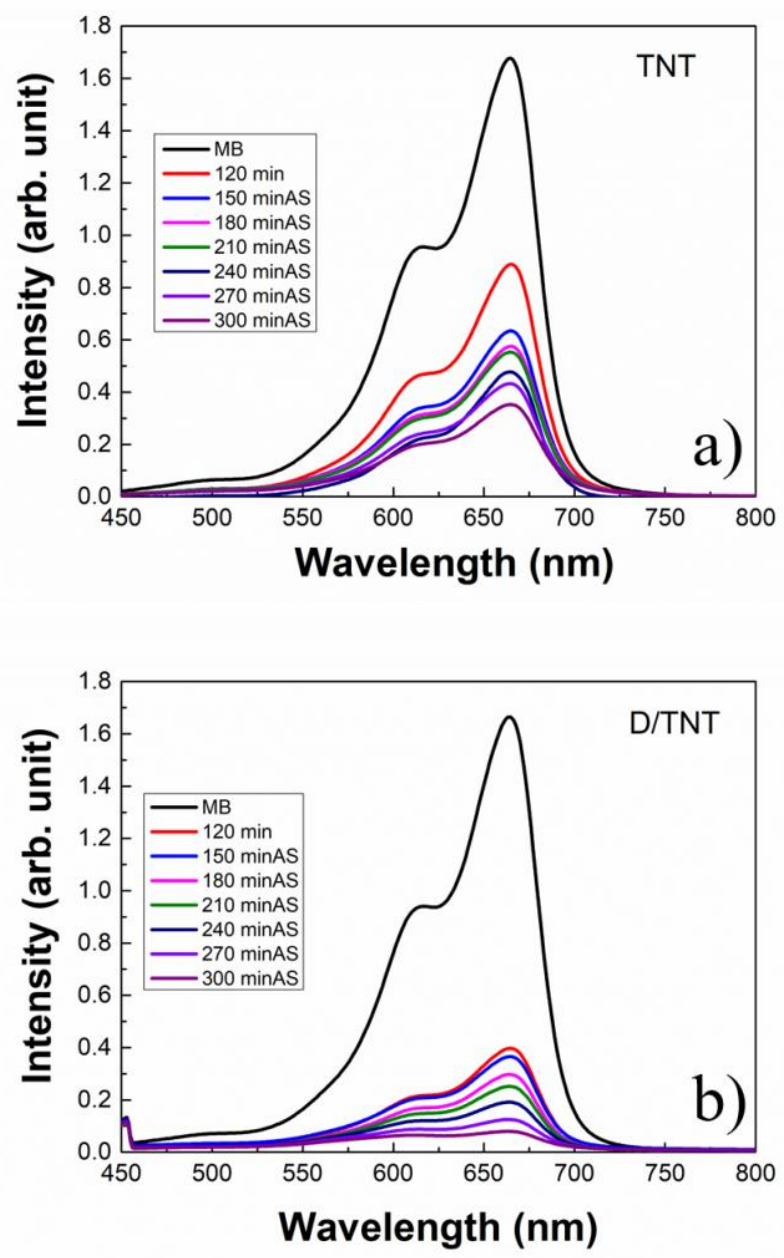

Figure 5. Sequence of physical adsorption and photocatalytic ability of materials with MB solution

Sunlight irradiation will help reduce the concentration of MB solution. Because natural sunlight had a broad wave range, this would help improve the ability to decompose organic compounds [5]. In the first stages of the photocatalytic process, the photocatalytic ability of TNT was higher than D/TNT composite. Specifically, in the first $30 \mathrm{~min}$, the photocatalytic ability was $29 \%$ and $8 \%$ for TNT and D/TNT composite, respectively (in Fig.5). However, at $90 \mathrm{~min}$, the photocatalytic ability was $38 \%$ and $37 \%$ for TNT and D/TNT composite, respectively, the performance of materials was similar (in Figure 5). At the end of photocatalytic process, the photocatalytic ability of D/TNT composite was higher than TNT. In particular, the performance was $61 \%$ and $80 \%$ for TNT and D/TNT composite, respectively (in Figure 5). Furthermore, D/TNT composite has good absorption ability, thus the amount of MB remaining is very low in the solution. Therefore, the amount of MB decomposed by D/TNT composite is less than the amount of MB decomposed by TNT in the photocatalytic process. Specifically, TNT and D/TNT composite decomposed $199 \mu \mathrm{mol} / 1$ and $119 \mu \mathrm{mol} / 1$ for MB in the solution, repectively. Hence, from the results of the photocatalytic process, the photocatalytic ability of TNT was improved after TNT composite with diatom. 


\subsection{Photoluminescene emission (PL)}

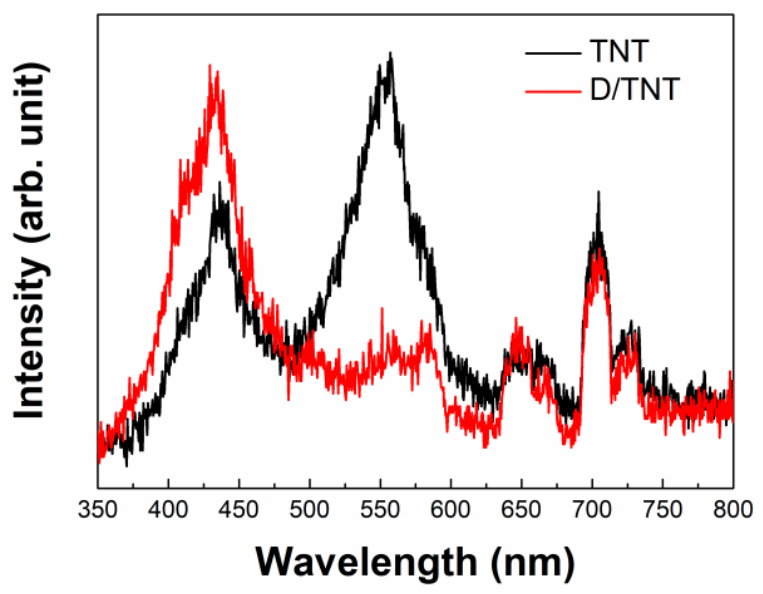

Figure 6. Room temperature photoluminescence emission spectra of TNT and D-TNT

Photoluminescence (PL) emission is a simple but powerful method to compare radiative recombination rate among samples through their normalized emission intensity [5]. Fig. 6 shows the normalized photoluminescence emission spectra of TNT and D/TNT composite. The PL intensity at $\sim 550 \mathrm{~nm}$ of D/TNT is lower than that of TNT, this peak is assigned to the radiative recombination of electrons in the conduction and the hole traps $\left(\mathrm{F}^{+}\right.$center $)$in the bandgap $[5,25]$. With lower intensity at this emission means that less radiative recombination rate, excited electrons have longer lifetime. In that case, they can decompose organic compounds through producing high oxidized groups such as superoxide and hydroxyl instead of recombination with trap holes in the bandgap. Thus, when TNT composited with diatom, the recombination rate is decreased thus enhancing photocatalytic ability.

\section{7. $E D X$}

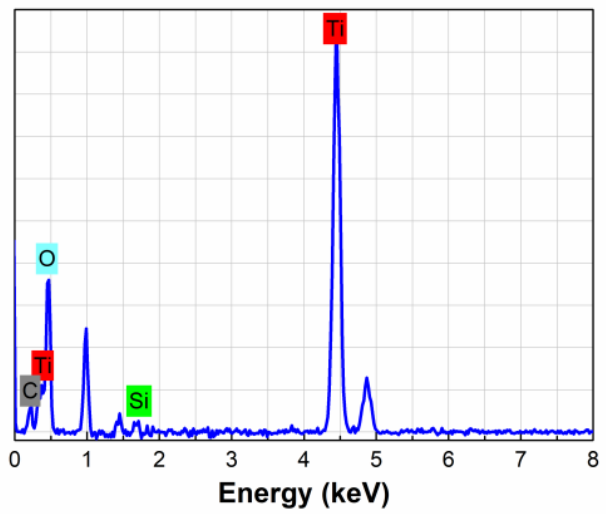

Figure 7. EDX spectrum of D/TNT composite. 
Fig. 7 shows the energy dispersive X-ray spectroscopy of D/TNT composite. According to result of EDX spectrum, the EDX of D/TNT composite has the appearance of Si and O element, this is due to the presence of diatom in the D/TNT composite [1]. The C peak assigned to organic matter on cell walls of diatom $[1,16]$. The weight and atomic of composition were shown in the table 1 .

Table 1. Shown weight and atomic of composition in D/TNT composite

\begin{tabular}{lll} 
Element & Mass $(\%)$ & Atom $(\%)$ \\
$\mathrm{O}$ & 43.02 & 69.61 \\
$\mathrm{Ti}$ & 55.32 & 29.91 \\
$\mathrm{Si}$ & 0.52 & 0.48 \\
Total & 98.86 & 100 \\
\hline
\end{tabular}

\section{Conclusion}

We successfully fabricated D/TNT composite by a facial hydrothermal method. The TEM and EDX results indicated the presence of diatom on TNT in the composite. TNT has a diameter of about $10 \mathrm{~nm}$ and a length of several hundred nanometers. In PL spectra, D/TNT has normalized intensity lower than TNT. This shows that the lifetime of excited electrons in D/TNT are longer than that of TNT, thus these excited electrons in the composite had more decomposition time which could improve the photocatalytic ability. Furthermore, the bandgap value was reduced from $3.55 \mathrm{eV}$ in pure TNT to $3.44 \mathrm{eV}$ in the composite, thereby, the enhancement of light absorption could induce better photocatalytic ability in D/TNT composite. As a result, MB degradation through the photocatalytic process was about $80 \%$ in D/TNT which is better than TNT (61\%).

\section{Acknowledgments}

This research is funded by the Vietnam National Foundation for Science and Technology Development (NAFOSTED) under grant number 103.02-2019.362

\section{References}

[1] L. Chetia, D. Kalita, G.A. Ahmed, Enhanced photocatalytic degradation by diatom templated mixed phase titania $\begin{array}{lllllll}\text { nanostructure, J. Photochem. Photobiol. A } & \text { Chem. } 338 & \text { (2017) }\end{array}$ https://doi.org/10.1016/j.jphotochem.2017.01.035

[2] N. Xuan Sang, N. Minh Quan, N. Huu Tho, N. Tri Tuan, T. Thanh Tung, Mechanism of enhanced photocatalytic activity of $\mathrm{Cr}$-doped $\mathrm{ZnO}$ nanoparticles revealed by photoluminescence emission and electron spin resonance, Semicond. Sci. Technol. 34 (2018) 025013. https://doi.org/10.1088/1361-6641/aaf820

[3] F. Jiang, S. Zheng, L. An, H. Chen, H. Effect of calcination temperature on the adsorption and photocatalytic activity of hydrothermally synthesized $\mathrm{TiO}_{2}$ nanotubes, Appl. Surf. Sci. 258 (2012) 7188-7194. https://doi.org/10.1016/j.apsusc.2012.04.032

[4] S.X. Nguyen, T. Thanh Tung, P.T. Lan Huong, N.H. Tho, D. Losic, Heterojunction of graphene and titanium dioxide nanotube composites for enhancing photocatalytic activity, J. Phys. D. Appl. Phys. 51 (2018) 265304. https://doi.org/10.1088/1361-6463/aac7ce

[5] N.X. Sang, P.T.L. Huong, T.T.M. Thy, P.T. Dat, V.C. Minh, N.H. Tho, Crystalline deformation and photoluminescence of titanium dioxide nanotubes during in situ hybridization with graphene: An example of the heterogeneous photocatalyst, $\quad$ Superlattices $\quad$ Microstruct. $\quad 121 \quad$ (2018) 9-15. https://doi.org/10.1016/j.spmi.2018.07.020

[6] P. Ferraro, E. De Tommasi, I. Rea, L. De Stefano, P. Dardano, G. Di Caprio, M.A. Ferrara, G. Coppola, M. Ritsch-Marte, S. Grilli, D. Stifter, Optics with diatoms: towards efficient, bioinspired photonic devices at the 
micro-scale, Optical Methods for Inspection, Characterization, and Imaging of Biomaterials 8792 (2013) 87920. https://doi.org/10.1117/12.2021613

[7] T. Fuhrmann, S. Landwehr, M. El Rharbi-Kucki, M. Sumper, Diatoms as living photonic crystals, Appl. Phys. B Lasers Opt. 78 (2004) 257-260. https://doi.org/10.1007/s00340-004-1419-4

[8] T.T. Nguyen, T.T. Tung, D. Losic, L.T. Lan Anh, L.H. Phuc, X.S. Nguyen, Electromigration with enhanced green emission in the titanium dioxide nanotube/graphene composite, Curr. Appl. Phys. 19 (2019) 1082-1087. https://doi.org/10.1016/j.cap.2019.06.008

[9] E. Van Eynde, Z.Y. Hu, T. Tytgat, S.W. Verbruggen, J. Watté, G. Van Tendeloo, I. Van Driessche, R. Blust, S. Lenaerts, Diatom silica-titania photocatalysts for air purification by bio-accumulation of different titanium sources, Environ. Sci. Nano. 3 (2016) 1052-1061. https://doi.org/10.1039/C6EN00163G

[10] Q. Chen, G.H. Du, S. Zhang, L. Peng, The structure of trititanate nanotubes, Acta Crystallographica Section B Structural Science 58 (2002) 587-593. https://doi.org/10.1107/S0108768102009084

[11] T. Kubo, A. Nakahira, A. Local structure of $\mathrm{TiO}_{2}$-derived nanotubes prepared by the hydrothermal process, J. Phys. Chem. C. 112 (2008) 1658-1662. https://doi.org/10.1021/jp076699d

[12] J. Yang, Z. Jin, X. Wang, W. Li, J. Zhang, S. Zhang, X. Guo, Z. Zhang, Study on composition, structure and formation process of nanotube $\mathrm{Na}_{2} \mathrm{Ti}_{2} \mathrm{O}_{4}(\mathrm{OH})_{2}$, Dalton Trans. 4 (2003) 3898-3901. https://doi.org/10.1039/B305585J

[13] D.V. Bavykin, V.N. Parmon, A.A. Lapkin, F.C. Walsh, The effect of hydrothermal conditions on the mesoporous structure of $\mathrm{TiO}_{2}$ nanotubes. J. Mater. Chem. 14 (2004) 3370-3377. https://doi.org/10.1039/B406378C

[14] M.Z. Wang, F.X. Liang, B. Nie, L.H. Zeng, L.X. Zheng, P. Lv, Y.Q. Yu, C. Xie, Y.Y. Li, L.B. Luo, TiO Nanotube Array/Monolayer Graphene Film Schottky Junction Ultraviolet Light Photodetectors, Part. Part. Syst. Char. 30 (2013) 630-636. https://doi.org/10.1002/ppsc.201300040

[15] L.C. Sim, K.H. Leong, S. Ibrahim, P. Saravanan, Graphene oxide and Ag engulfed $\mathrm{TiO}_{2}$ nanotube arrays for enhanced electron mobility and visiblelight-driven photocatalytic performance, J. Mater. Chem. A 2 (2014) 5315-5322. https://doi.org/10.1039/C3TA14857B

[16] M.S. Aw, S. Simovic, J. Addai-Mensah, D. Losic, Silica microcapsules from diatoms as new carrier for delivery of therapeutics, Nanomedicine 6 (2011) 1159-1173. https://doi.org/10.2217/nnm.11.29

[17] F.D. Hardcastle, Raman Spectroscopy of Titania $\left(\mathrm{TiO}_{2}\right)$ Nanotubular Water-Splitting Catalysts, Journal of the Arkansas Academy of Science 65 (2011). https://scholarworks.uark.edu/jaas/vol65/iss1/9/

[18] L. Qian, Z.L. Du, S.Y. Yang, Z.S. Jin, S. Raman study of titania nanotube by soft chemical process, J. Mol. Struct. 749 (2005) 103-107. https://doi.org/10.1016/j.molstruc.2005.04.002

[19] S.H. Byeon, S.O. Lee, H. Kim, Structure and Raman Spectra of Layered Titanium Oxides, J. Solid State Chem. 130 (1997) 110-116. https://doi.org/10.1006/jssc.1997.7286

[20] P.C. Sevinc, X. Wang, Y. Wang, D. Zhang, A.J. Meixner, H.P. Lu, Simultaneous spectroscopic and topographic near-field imaging of $\mathrm{TiO}_{2}$ single surface states and interfacial electronic coupling, Nano Lett. 11 (2011) 14901494. https://doi.org/10.1021/nl104160n

[21] T K. Thiyagarajan, S. Samuel, S.G. Babu, NiO/ $\mathrm{C}_{3} \mathrm{~N}_{4}$ : hybrid photocatalyst for the enhanced photodegradation of organic pollutant under visible light, Mater. Res. Express 5 (2018) 115503. https://doi.org/10.1088/20531591/aadc3e

[22] A. Sáenz-Trevizo, P. Amézaga-Madrid, P. Pizá-Ruiz, W. Antúnez-Flores, M. Miki-Yoshida, Optical Band Gap Estimation of ZnO Nanorods, Mater. Res. 19 (2016) 33-38. https://doi.org/10.1590/1980-5373-mr-2015-0612

[23] R. López, R. Gómez, Band-gap energy estimation from diffuse reflectance measurements on sol-gel and commercial $\mathrm{TiO}_{2}$ : A comparative study, J. Sol-Gel Sci. Tech. 61 (2012) 1-7. https://doi.org/10.1007/s10971-0112582-9

[24] Q. Zhang, N. Bao, X. Wang, X. Hu, X. Miao, M. Chaker, D. Ma, Advanced Fabrication of Chemically Bonded Graphene/ $/ \mathrm{TiO}_{2}$ Continuous Fibers with Enhanced Broadband Photocatalytic Properties and Involved Mechanisms Exploration, Sci. Rep. 6 (2016) 1-15. https://doi.org/10.1038/srep38066

[25] C.C. Mercado, F.J. Knorr, J.L. McHale, S.M. Usmani, A.S. Ichimura, L.V. Saraf, Location of hole and electron traps on nanocrystalline anatase $\mathrm{TiO}_{2}, \quad$ J. Phys. Chem. C 116 (2012) 10796-10804. https://doi.org/10.1021/jp301680d. 